\title{
RECURSOS DESENVOLVIMENTAIS E DESENVOLVIMENTO JUVENIL POSITIVO (PYD): UMA REVISÃO
}

\author{
DEVELOPMENTAL RESOURCES AND POSITIVE YOUTH DEVELOPMENT (PYD): A \\ REVIEW
}

\author{
Carla Assis dos Santos Melo ${ }^{1}$ \\ Islane Cristina Martins ${ }^{2}$
}

RESUMO: Introdução: Independente da condição socioeconômica, os jovens estão sujeitos às situações de risco e o desenvolvimento positivo da juventude - PYD - priorizando a integralidade do jovem, busca potencializar suas capacidades combinando com os recursos desenvolvimentais. Objetivo: Objetivou-se realizar uma revisão integrativa da literatura a fim de discutir os recursos desenvolvimentais e PYD. Materiais e Métodos: Buscou-se referências nas bases Periódicos CAPES e Google Scholar em setembro de 2020. A investigação permitiu identificar 27 artigos que se adequaram aos critérios estabelecidos. Resultados: Observou-se que ioo\% dos artigos mencionaram ao menos um dos 40 recursos de desenvolvimento do modelo de Benson comprovando intensa relação entre tais recursos desenvolvimentais e PYD. Conclusão: Tanto os recursos desenvolvimentais (criatividade, autoeficácia, estrutura escolar, vínculos familiares, etc.) quanto PYD, baseado em princípios que contemplam todo o ser do jovem, suas capacidades e forças, são proposições que dialogam entre si, promovendo comportamentos positivos e o próprio bem-estar.

Palavras-chaves: Comportamentos. Desenvolvimento Juvenil Positivo (PYD). Fatores de risco. Oportunidades. Prevenção.

ABSTRACT: Introduction: Regardless of socioeconomic status, young people are subject to risky situations and the positive development of youth - PYD - prioritizing the integrality of young people, seeks to enhance their capabilities combining with developmental resources. Objective: The objective was to conduct an integrative literature review in order to discuss developmental resources and PYD. Materials and Methods: References were sought in the CAPES and Google Scholar Periodical databases in September 2020. The investigation identified 27 articles that met the established criteria. Results: It was observed that $100 \%$ of the articles mentioned at least one of the 40 development resources of the Benson model, proving an intense relationship between such

1 Possui graduação em Administração pelo Instituto Federal da Bahia (2009), graduação em Ciências Sociais pela Universidade Federal da Bahia (2015), graduação em Pedagogia pela UNINTER (2018) e especialização em Tutoria e Educação à Distância pela Faculdade Jardins (2018). Atualmente, é coordenadora pedagógica (servidora pública) em uma unidade escolar da Secretaria da Educação do Estado da Bahia - SEC. Email: carla.assis@enova.educacao.ba.gov.br.

${ }^{2}$ Biomédica pela Universidade Federal de Pernambuco-PE, mestre em Neurociências pelo Programa de PósGraduação em Neuropsiquiatria e Ciências do Comportamento - UFPE e doutoranda pelo PPGBAS LIKAUFPE. Professional and Self Coach - IBC. Já auxiliou mais de 500 pessoas por meio de assessorias científicas a publicarem seus artigos e serem aprovados em mestrados e doutorados nas áreas de humanas, exatas e saúde de Instituições Federais brasileiras. E-mail: relacionamento@passenomestrado.com. 
developmental resources and PYD. Conclusion: Both the developmental resources (creativity, selfefficacy, school structure, family bonds, etc.) and PYD, based on principles that contemplate the whole being of the young person, their capacities and strengths, are propositions that dialogue with each other, promoting behaviors positives and well-being.

Keywords: Behaviors. Positive Youth Development (PYD). Risk factors. Opportunities, Prevention.

\section{INTRODUÇÃO}

O desenvolvimento positivo da juventude (PYD) é uma filosofia holística que prioriza aspectos físicos, pessoais, sociais, emocionais, intelectuais e espirituais do público jovem, priorizando o seu desenvolvimento saudável (Durlak et al., 2007).

Segundo Franco e Rodrigues (2018), PYD elucida proporcionar a saúde de todos os jovens, tendo em vista potencializar suas capacidades e enfatizar as características positivas desse período, ajustadas com recursos do ambiente e de pessoas significativas.

Dito isso, surge, em 1990, o conceito de Recursos Desenvolvimentais proposto pelo Search Institute buscando apresentar o que os jovens precisam para um desenvolvimento bem-sucedido (Benson 2006, 2007 citado por Soares, Pais-Ribeiro \& Silva, 2018, p.3).

Desse modo, o PYD está relacionado com a prevenção a fatores de risco que são condições associadas à alta probabilidade de ocorrência de resultados negativos ou indesejáveis, de acordo com Reppold et al. (2002 citado por Maia \& Williams 2005, p.92).

Da mesma forma, existem relações empíricas entre prevenção de inúmeros comportamentos negativos entre jovens - uso e abuso de álcool e outras drogas, violência - e o desenvolvimento positivo, conforme os resultados da pesquisa de Eichas, Wreder e Olsson (2019).

Nesse sentido, dentre recursos desenvolvimentais, muitos estudos evidenciam que intervenções em algumas competências fomentam a prevenção de problemas comportamentais em todas as etapas da vida da pessoa, inclusive na juventude para Dutra-Thomé et al. (2017).

Ainda, segundo Dutra-Thomé et al. (2017), essas competências de relevância sociomemocional podem ser a autoeficácia e a autoestima, considerando que são fatores de proteção que são também dispositivos do PYD.

Contudo, mais que prevenção ou correção de problemas, PYD busca resultados positivos, já que, é um processo de desenvolvimento sustentado no pacto de permitir que os jovens prosperem, pois todos têm potencialidades, conforme Hamilton M. A., Hamilton S. F., Pittman (2004).

Por isso, para aprofundar PYD, é relevante compreender sobre o comportamento humano que se traduz num conjunto de habilidades sociais e pensamentos que interpretam os contextos sociais nos quais pessoas interagem e articulam (Pereira, Dutra-Thomé e Koller, 2016). 
Portanto, PYD possibilita a promoção de ambientes sustentáveis com ações para jovens que podem prevenir comportamentos de risco, como violência, evasão escolar, uso de álcool e outras drogas para Senna e Dessen (2012 apud Franco e Rodrigues 2018, p.2269).

Também, o modelo de Recursos do Desenvolvimento tem demonstrado ser influente para o desenvolvimento bem-sucedido do jovem, segundo Benson, Scales \& Syvertsen (2oII citado por Soares et al., 2018, p.13), inclusive, em diferentes contextos socioeconômicos.

Conforme Fundo de População das Nações Unidas (Agência Brasil, 2014), quase um terço dos jovens entre ro e 24 anos no mundo vive em situação de vulnerabilidade social. Independente da condição socioeconômica, até na Adultez Emergente (AE), fase entre adolescência e vida adulta com comportamentos que postergam obrigação de adotar papéis adultos como formação familiar e independência financeira dos pais (Arnet, 2011 como citado em Pereira et al., 2016, p.269), os jovens estão sujeitos às situações de risco, pois diante das incertezas vivenciam sentimentos negativos e instabilidade (Pereira et al., 2016).

Para tanto, o objetivo do presente estudo foi realizar uma revisão integrativa da literatura a fim de discutir os recursos desenvolvimentais e o desenvolvimento juvenil positivo (PYD).

\section{Materiais e métodos}

Em setembro de 2020, foi realizado um levantamento da literatura nas bases de dados Periódicos CAPES e Google Acadêmico. As palavras-chave utilizadas foram as seguintes: “Desenvolvimento Juvenil Positivo (PYD) " AND "fatores de risco" AND "prevenção" AND "comportamentos" AND "oportunidades" AND "Positive Youth Development (PYD)" AND "risk factors" AND "prevention" AND "behaviors" AND "opportunities" em ambas as bases de dados. Foram selecionados 27 artigos sendo incluídos segundo os critérios de elegibilidade conforme a Figura I. Os critérios de inclusão foram: artigos nos idiomas Espanhol, Inglês e Português, nos últimos cinco anos, envolvendo recursos desenvolvimentais e desenvolvimento juvenil positivo (PYD). 
Figura 1. Fluxograma e critérios de seleção e inclusão
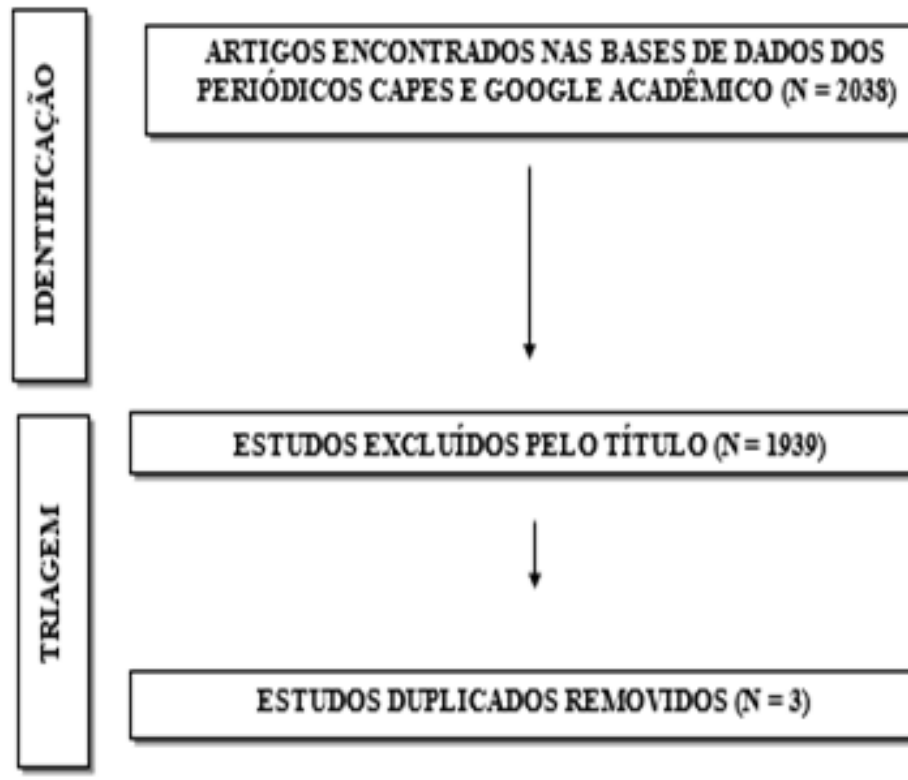

ESTUDOS EXCL UIDOS PELO TÍTULO $(\mathrm{N}=1939)$
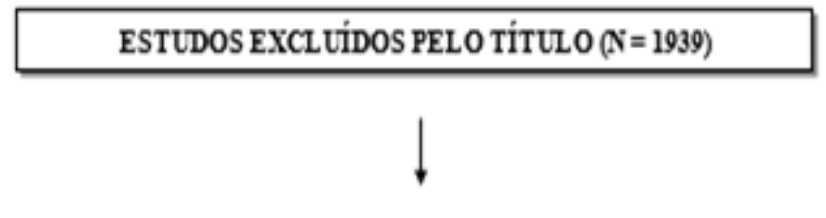

ESTUDOS DUPLICADOS REMOVIDOS $(N=3)$
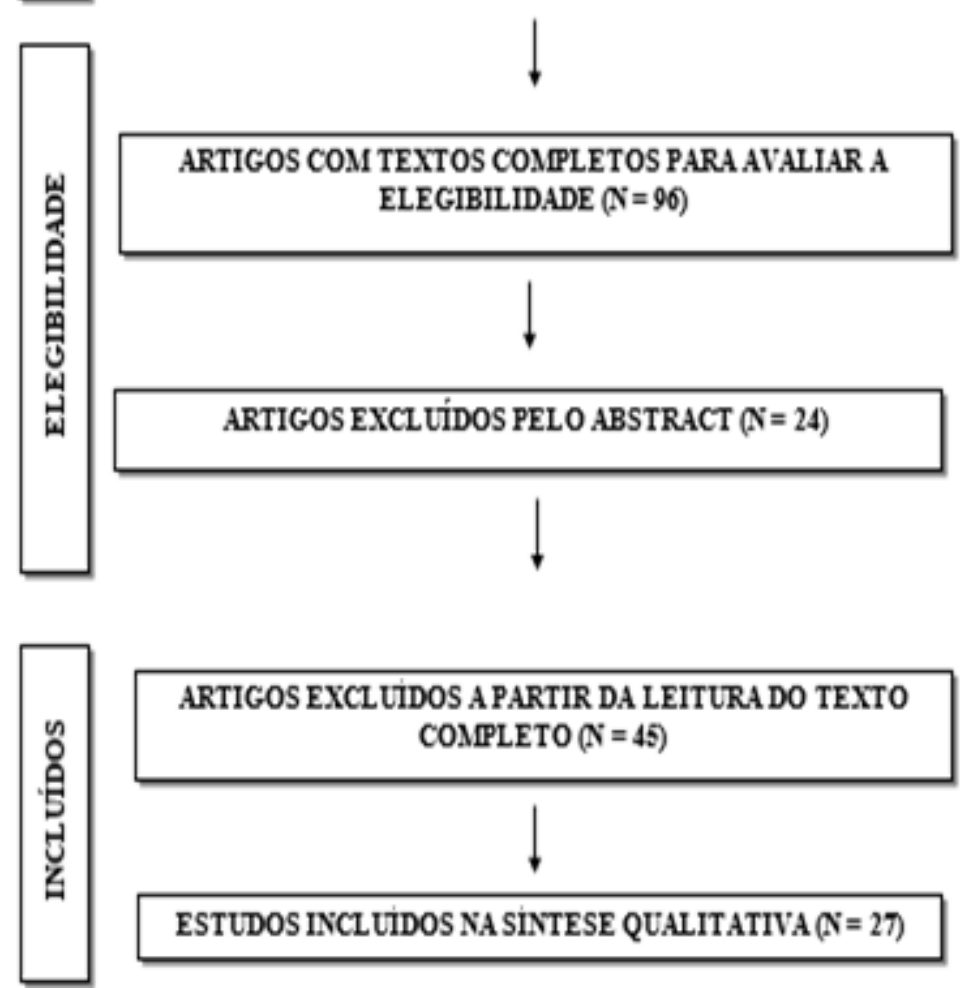

ARTIGOS EXCLUIDOS A PARTIR DA LEITURA DO TEXTO COMPLETO $(N=45)$

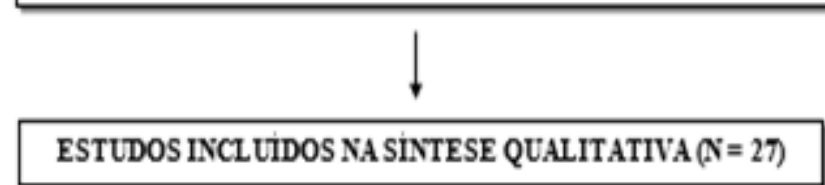

I. Resultados

Os resultados do presente estudo encontram-se na Tabela I 
Revista Ibero- Americana de Humanidades, Ciências e Educação- REASE $\quad$ open ${ }_{\text {Access }}$

Tabela I - Demonstrativo dos artigos que integram a Revisão.

\begin{tabular}{|c|c|c|c|c|c|c|}
\hline$\# \mathbf{N}$ & Data & Título & Autores & Periódico & Objetivos & Resultados \\
\hline I & 2020 & $\begin{array}{l}\text { The prediction of } \\
\text { depression based on } \\
\text { religious coping and } \\
\text { the components of } \\
\text { positive youth } \\
\text { development in } \\
\text { adolescents }\end{array}$ & $\begin{array}{l}\text { Mohammad } \\
\text { Sadegh Sarizadeh, } \\
\text { Mahmoud Najafi \& } \\
\text { Ali Mohammad } \\
\text { Rezaei. }\end{array}$ & $\begin{array}{l}\text { Mental Health, } \\
\text { Religion \& Culture }\end{array}$ & $\begin{array}{l}\text { Prevenir a depressão } \\
\text { mediante enfrentamento } \\
\text { religioso e programas de } \\
\text { desenvolvimento positivo } \\
\text { da juventude } \\
\text { adolescentes. }\end{array}$ & $\begin{array}{l}\text { Os resultados revelaram que os } \\
\text { programas PYD, assim como, } \\
\text { programas de enfretamento } \\
\text { religioso e espiritual, são táticas } \\
\text { eficazes para superar a depressão e } \\
\text { elevar o bem-estar de adolescentes. }\end{array}$ \\
\hline 2 & 2020 & $\begin{array}{l}\text { Positive } \\
\text { Development } \\
\text { Through Leisure: } \\
\text { Confronting the } \\
\text { COVID-ig Pandemic }\end{array}$ & $\begin{array}{l}\text { Andrea Vest } \\
\text { Etteka, Jennifer P. } \\
\text { Agans. }\end{array}$ & $\begin{array}{l}\text { Journal of Youth } \\
\text { Development }\end{array}$ & $\begin{array}{l}\text { Realizar uma revisão dos } \\
\text { elementos-chave que } \\
\text { contribuem para a eficácia } \\
\text { dos programas de } \\
\text { desenvolvimento positivo } \\
\text { juvenil e sugerir formas de } \\
\text { sustentar, adaptar e criar } \\
\text { esses elementos e trazê-los } \\
\text { para ambientes de lazer não } \\
\text { estruturados durante a } \\
\text { pandemia. }\end{array}$ & $\begin{array}{l}\text { Foi possível identificar desafios } \\
\text { específicos para a implementação, } \\
\text { destacar exemplos inovadores de } \\
\text { adaptação exibidos nas } \\
\text { comunidades afetadas. Conclui-se } \\
\text { que existe a necessidade de ações } \\
\text { para apoiar programas PYD por } \\
\text { meio do lazer, mesmo em face de } \\
\text { crise. }\end{array}$ \\
\hline 3 & 2020 & $\begin{array}{l}\text { A Systematic Review } \\
\text { of Program Quality in } \\
\text { the Field of Positive } \\
\text { Youth Development }\end{array}$ & $\begin{array}{l}\text { Jeantyl Norze, } \\
\text { Melissa Cater. }\end{array}$ & $\begin{array}{l}\text { Advances in Sciences } \\
\text { and Humanities - } \\
\text { Science Publishing } \\
\text { Group }\end{array}$ & $\begin{array}{l}\text { Avaliar as áreas de } \\
\text { competência no que diz } \\
\text { respeito à programação de } \\
\text { qualidadł3 zque abordam o } \\
\text { desenvolvimento positivo } \\
\text { da juventude. }\end{array}$ & $\begin{array}{l}\text { Os resultados assinalaram que } \\
\text { existem áreas-chave onde a } \\
\text { qualidade do programa que abrange } \\
\text { o desenvolvimento da juventude } \\
\text { pode acontecer de maneira eficaz } \\
\text { pelo treinamento de pessoal. } \\
\text { Observou-se que poucas pesquisas } \\
\text { têm se debruçado nas relações entre } \\
\text { o treinamento do pessoal e a } \\
\text { qualidade do programa. }\end{array}$ \\
\hline 4 & 2020 & $\begin{array}{l}\text { Positive youth } \\
\text { development as an } \\
\text { improvement resource }\end{array}$ & $\begin{array}{l}\text { Catherine S. } \\
\text { Kramer, Kristen C. } \\
\text { Wilcox \& Hal A. } \\
\text { Lawson. }\end{array}$ & $\begin{array}{lr}\text { Taylor \& } & \text { Francis } \\
\text { Online - Prevenção ao } \\
\text { Fracasso } & \text { Escolar: } \\
\text { Educação } & \text { Alternativa }\end{array}$ & $\begin{array}{l}\text { Analisar as práticas } \\
\text { educacionais dos professores } \\
\text { das escolas de ensino médio } \\
\text { chamadas de "batedores de }\end{array}$ & $\begin{array}{l}\text { Os resultados revelaram que essas } \\
\text { escolas são caracterizadas por } \\
\text { educadores que priorizam práticas }\end{array}$ \\
\hline
\end{tabular}


Revista Ibero- Americana de Humanidades, Ciências e Educação- REASE open access $^{\text {acto }}$

\begin{tabular}{|c|c|c|c|c|c|c|}
\hline & & $\begin{array}{l}\text { in odds-beating } \\
\text { secondary schools }\end{array}$ & & $\begin{array}{l}\text { para Crianças e } \\
\text { Jovens }\end{array}$ & $\begin{array}{l}\text { chances", ou seja, escolas } \\
\text { modelos que obtém uma } \\
\text { graduação melhor do que os } \\
\text { resultados previstos entre os } \\
\text { diversas representações } \\
\text { sociais desfavorecidos } \\
\text { economicamente. }\end{array}$ & $\begin{array}{l}\text { do desenvolvimento positivo de } \\
\text { jovens (PYD). }\end{array}$ \\
\hline 5 & 2019 & $\begin{array}{l}\text { Positive } \quad \text { youth } \\
\text { development: current } \\
\text { perspectives }\end{array}$ & $\begin{array}{l}\text { Daniel TL Shek, } \\
\text { Diya Dou, Xiaoqin } \\
\text { Zhu, Wenyu Chai. }\end{array}$ & $\begin{array}{l}\text { Adolescent Health, } \\
\text { Medicine } \\
\text { Therapeutics }\end{array}$ & $\begin{array}{l}\text { Revisar a literatura das } \\
\text { perspectivas } \\
\text { contemporâneas sobre o } \\
\text { desenvolvimento positivo } \\
\text { da juventude (PYD). }\end{array}$ & $\begin{array}{l}\text { Os resultados apontaram que } \\
\text { existe uma ausência de integração } \\
\text { entre os processos, filosofias e } \\
\text { programas do PYD e por } \\
\text { consequência não há certezas na } \\
\text { compreensão de como } \\
\text { implementar o PYD. Foi possível } \\
\text { observar também que os diferentes } \\
\text { modelos PYD buscam atingir } \\
\text { diferentes resultados, o que gera } \\
\text { dificuldade nas comparações entre } \\
\text { os estudos. }\end{array}$ \\
\hline \multicolumn{7}{|c|}{ Continua } \\
\hline 6 & 2019 & $\begin{array}{l}\text { Adolescence and } \\
\text { creativity: cognitions } \\
\text { and affect involved in } \\
\text { positive youth } \\
\text { development }\end{array}$ & $\begin{array}{l}\text { Claire de } \\
\text { Mézerville. }\end{array}$ & $\begin{array}{l}\text { Arts and Humanities } \\
\text { Open Access Journal }\end{array}$ & $\begin{array}{l}\text { Investigar e expor a } \\
\text { criatividade como uma } \\
\text { habilidade imprescindível } \\
\text { para a educação na } \\
\text { adolescência. } \\
\text { 333 }\end{array}$ & $\begin{array}{l}\text { Constatou-se que a criatividade é } \\
\text { uma habilidade para a } \\
\text { autorrealização e vinculação } \\
\text { positiva na sociedade e que para ser } \\
\text { desenvolvida exige conjunturas } \\
\text { escolares e uma rede de apoio } \\
\text { comprometida em promover o } \\
\text { desenvolvimento positivo da } \\
\text { juventude e incentivar os } \\
\text { adolescentes a se envolverem, a } \\
\text { criare prosperar. }\end{array}$ \\
\hline 7 & 2019 & $\begin{array}{l}\text { Indicators of positive } \\
\text { youth development } \\
\text { can be maladaptive: } \\
\text { The example case of } \\
\text { caring }\end{array}$ & $\begin{array}{l}\text { G. John Geldhofa, } \\
\text { Torill Larsenb, } \\
\text { Helga } \quad \text { Urkeb, } \\
\text { Ingrid Holsenb, } \\
\text { Hillary Lewisa , } \\
\text { Corine P. Tylera. }\end{array}$ & Journal of Adolescence & $\begin{array}{l}\text { Enfatizar que a possibilidade } \\
\text { de medidas individuais de } \\
\text { desenvolvimento adaptativo } \\
\text { pode refletir, em algumas } \\
\text { realidades, }\end{array}$ & $\begin{array}{l}\text { Os resultados apresentaram que } \\
\text { pode haver um desequilíbrio entre } \\
\text { os Cinco Cs do Desenvolvimento } \\
\text { Positivo da Juventude, pois as } \\
\text { associações ao bem-estar em } \\
\text { medidas não equilibradas podem }\end{array}$ \\
\hline
\end{tabular}


Revista Ibero- Americana de Humanidades, Ciências e Educação- REASE open $\odot$ access

\begin{tabular}{|c|c|c|c|c|c|c|}
\hline & & & & & $\begin{array}{l}\text { condicionamentos de } \\
\text { desenvolvimento negativo. }\end{array}$ & $\begin{array}{l}\text { ser associadas a sintomas } \\
\text { depressivos, podendo ocorrer que } \\
\text { em certas situações o que deveria } \\
\text { ser "mais" é na verdade "menos". }\end{array}$ \\
\hline 8 & 2019 & $\begin{array}{l}\text { The Role of Positive } \\
\text { Youth Development, } \\
\text { Educational Policy, } \\
\text { and Cultural } \\
\text { Relevancy in School } \\
\text { Settings }\end{array}$ & $\begin{array}{l}\text { Rolanda L. Ward, } \\
\text { Tanyetta Carter, } \\
\text { and Nazia Siddiq. }\end{array}$ & $\begin{array}{l}\text { Artigo publicado pelo } \\
\text { Departamento de } \\
\text { Serviço } \\
\text { Niagara University, } \\
\text { O'Shea Hall, Lower } \\
\text { Level, } \\
\text { University }\end{array}$ & $\begin{array}{l}\text { Relacionar crescimento, } \\
\text { promoção das teorias do } \\
\text { desenvolvimento positivo } \\
\text { da juventude (PYD) e } \\
\text { implementação nas atuais } \\
\text { transformações na política } \\
\text { federal que apoiam a } \\
\text { inclusão da juventude na } \\
\text { reforma escolar. Promover } \\
\text { um debate sobre a } \\
\text { programação histórica do } \\
\text { PYD, papel da relevância } \\
\text { cultural em ambientes } \\
\text { educacionais e futuras áreas } \\
\text { de pesquisa PYD no nível } \\
\text { escolar. }\end{array}$ & $\begin{array}{l}\text { Os resultados indicaram que } \\
\text { oportunidades para jovens iniciar, } \\
\text { projetar, implementar e avaliar } \\
\text { programas e intervenções distritais } \\
\text { são favoráveis, não somente ao } \\
\text { desenvolvimento inerente de cada } \\
\text { jovem, como também às } \\
\text { comunidades escolares nas quais } \\
\text { passam a maior parte do tempo. }\end{array}$ \\
\hline 9 & 2019 & $\begin{array}{l}\text { Contributions } \\
\text { of Positive Youth } \\
\text { Development } \\
\text { to Intervention } \\
\text { Science }\end{array}$ & $\begin{array}{l}\text { Kyle Eichas, } \\
\text { Laura Ferrer- } \\
\text { Wreder, } \\
\text { Tina M. Olsson. }\end{array}$ & $\begin{array}{l}\text { Child \& Youth Care } \\
\text { Forum }\end{array}$ & $\begin{array}{l}\text { Destacar as principais } \\
\text { contribuições do PYD para a } \\
\text { identificação de modelos } \\
\text { mais completos de } \\
\text { intervenção juvenil, como } \\
\text { também para apontar } \\
\text { direções para futuras } \\
\text { pesquisas de intervenção } \\
\text { PYD. 334 }\end{array}$ & $\begin{array}{l}\text { Os resultados mostraram } \\
\text { vantagens práticas na compreensão } \\
\text { de relações empíricas entre PYD, } \\
\text { tratamento e prevenção no } \\
\text { caminho para alcançar mais } \\
\text { pesquisa de intervenção totalmente } \\
\text { integrada, bem como desafios } \\
\text { metodológicos envolvidos na busca } \\
\text { de uma agenda. }\end{array}$ \\
\hline
\end{tabular}


Revista Ibero- Americana de Humanidades, Ciências e Educação- REASE open $\odot$ Access

\begin{tabular}{|c|c|c|c|c|c|c|}
\hline IO & 2018 & $\begin{array}{l}\text { The Evolution of } \\
\text { Positive Youth } \\
\text { Development as a Key } \\
\text { International } \\
\text { Development } \\
\text { Approach }\end{array}$ & Christina Olenik & Global Social Welfare & $\begin{array}{l}\text { Investigar sobre a evolução } \\
\text { do PYD no } \\
\text { desenvolvimento } \\
\text { internacional e sobre as } \\
\text { evidências de PYD em } \\
\text { países de baixa e média } \\
\text { renda. }\end{array}$ & $\begin{array}{l}\text { Os resultados identificaram } \\
\text { doadores interessados em incluir a } \\
\text { juventude em seu próprio } \\
\text { planejamento e estratégia } \\
\text { organizacional, bem como em } \\
\text { garantir que os jovens tenham voz } \\
\text { forte na política e na tomada de } \\
\text { decisões a nível da comunidade e } \\
\text { do país. Observou-se que, nos } \\
\text { últimos anos, muitos dos principais } \\
\text { doadores no desenvolvimento } \\
\text { internacional estabeleceram } \\
\text { políticas ou estratégias para a } \\
\text { juventude indicando a existência } \\
\text { de uma oportunidade para } \\
\text { construir a prática e medição do } \\
\text { PYD. }\end{array}$ \\
\hline II & 2018 & $\begin{array}{l}\text { Positive } \\
\text { Development } \\
\text { Sustainability } \\
\text { (PYDSS): Scale } \\
\text { Development of an } \\
\text { Assessment Tool }\end{array}$ & $\begin{array}{l}\text { Michael Sieng, } \\
\text { Scott Cloutier, } \\
\text { Katherine Irimata. }\end{array}$ & $\begin{array}{l}\text { Journal of Social } \\
\text { Change }\end{array}$ & $\begin{array}{l}\text { Detalhar o desenvolvimento } \\
\text { da Escala Positiva de } \\
\text { Sustentabilidade do } \\
\text { Desenvolvimento Juvenil e } \\
\text { a ferramenta de autorrelato } \\
\text { para avaliar os impactos dos } \\
\text { programas de PYD. }\end{array}$ & $\begin{array}{l}\text { Os resultados indicam que diversas } \\
\text { quaetões devem ser retiradas para } \\
\text { garantir um maior ajuste do } \\
\text { modelo para ambos os conjuntos de } \\
\text { dados e suporte para noção de } \\
\text { aplicação global; Verificou-se que } \\
\text { existe a estrutura de medição } \\
\text { original não se aplica a todos os } \\
\text { jovens em todos os países, visto } \\
\text { que, cada cultura e cada programa } \\
\text { PYD são diferentes. }\end{array}$ \\
\hline 12 & 2018 & $\begin{array}{lr}\text { Understanding } & \text { the } \\
\text { impact of youth } \\
\text { engagement } \\
\text { positive } \\
\text { development }\end{array}$ & Kristy Allen & $\begin{array}{l}\text { Dissertação para } \\
\text { cumprimento parcial } \\
\text { dos requisitos para o } \\
\text { Grau de Mestre em } \\
\text { Saúde Pública / BA } \\
\text { (Hons) Psychology, } \\
\text { University of Guelph, } \\
\text { 2or8 / Faculty of } \\
\text { Health Sciences. }\end{array}$ & $\begin{array}{l}\text { Analisar ações de } \\
\text { engajamento dos jovens para } \\
\text { promover o bem-estar } \\
\text { juvenil utilizando o PYD } \\
\text { dentro de um contexto } \\
\text { canadense. }\end{array}$ & $\begin{array}{l}\text { A pesquisa resultou em } 35 \text { artigos } \\
\text { analisados e diversos elementos de } \\
\text { ações para engajamento da } \\
\text { juventude foram associados a } \\
\text { resultados positivos de curto e } \\
\text { longo prazo que considerou a } \\
\text { juventude em vários domínios. }\end{array}$ \\
\hline
\end{tabular}


Revista Ibero- Americana de Humanidades, Ciências e Educação- REASE $\quad$ open $○$ Access

\begin{tabular}{|c|c|c|c|c|c|c|}
\hline 13 & 2018 & $\begin{array}{lr}\text { Autoeficácia } & \text { e } \\
\text { Desenvolvimento } \\
\text { Positivo dos Jovens: } \\
\text { Uma } & \text { Revisão } \\
\text { Narrativa } & \text { da } \\
\text { Literatura } & \end{array}$ & $\begin{array}{l}\text { Gisele de Rezende } \\
\text { Franco, Marisa } \\
\text { Cosenza Rodrigues. }\end{array}$ & $\begin{array}{l}\text { Trends in Psychology } \\
\text { / Temas em Psicologia }\end{array}$ & $\begin{array}{l}\text { Realizar uma revisão que } \\
\text { busca discutir relações entre } \\
\text { crenças de autoeficácia e } \\
\text { recursos desenvolvimentais } \\
\text { em diferentes domínios } \\
\text { sociais. }\end{array}$ & $\begin{array}{l}\text { Identificou-se que, isoladamente, } \\
\text { autoeficácia e desempenho } \\
\text { acadêmico contam com produções } \\
\text { científicas mais expressivas, } \\
\text { contudo a associação entre essas } \\
\text { variáveis requer mais pesquisas. } \\
\text { Verificou-se que pesquisas } \\
\text { associando a autoeficácia aos } \\
\text { recursos do PYD são recentes, } \\
\text { existe predominantemente } \\
\text { pesquisas internacionais e poucas } \\
\text { no contexto, sobretudo, brasileiro. }\end{array}$ \\
\hline I4 & 2017 & $\begin{array}{l}\text { Parental and Peer } \\
\text { Attachment and its } \\
\text { Relationship with } \\
\text { Positive } \\
\text { Development }\end{array}$ & $\begin{array}{lr}\text { Nor } & \text { Hidayah } \\
\text { Mohamed, } & \text { Siti } \\
\text { Raba'ah Hamzah, } \\
\text { Ismi Arif Ismail } \\
\text { Bahaman } \\
\text { Samah. }\end{array}$ & $\begin{array}{l}\text { International Journal } \\
\text { of Academic Research } \\
\text { in Business and Social } \\
\text { Sciences }\end{array}$ & $\begin{array}{l}\text { Pesquisar a relação entre o } \\
\text { afeto dos pais e dos pares no } \\
\text { desenvolvimento juvenil } \\
\text { usando os } 5 \text { Cs no Modelo de } \\
\text { PYD. }\end{array}$ & $\begin{array}{l}\text { O resultado apresentou que há uma } \\
\text { relação relevante entre os dois } \\
\text { fatores e PYD. O estudo mostrou } \\
\text { que o apego dos pais e dos pares } \\
\text { estava contribuindo com fatores } \\
\text { para o desenvolvimento positivo } \\
\text { da juventude. }\end{array}$ \\
\hline \multicolumn{7}{|l|}{ Continua } \\
\hline is & 2017 & $\begin{array}{l}\text { Youth identity from } \\
\text { ecological perspective } \\
\text { between education } \\
\text { and prevention }\end{array}$ & $\begin{array}{l}\text { Jerzy Nikitorowicz } \\
\text { Krzysztof Sawicki } \\
\text { Emilia Zylkiewicz- } \\
\text { Plonska. }\end{array}$ & $\begin{array}{l}\text { Society. Integration. } \\
\text { Education }\end{array}$ & 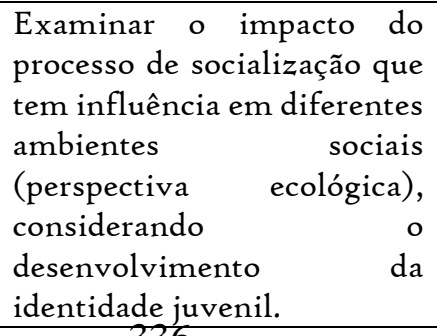 & $\begin{array}{l}\text { Os resultados apresentaram que a } \\
\text { eficácia das ações realizadas a nível } \\
\text { local depende em grande medida do } \\
\text { conhecimento e experiência } \\
\text { adquiridos e de procedimentos de } \\
\text { avaliação implementados. }\end{array}$ \\
\hline 16 & 2017 & $\begin{array}{l}\text { Exploring the effect of } \\
\text { autonomous student } \\
\text { experiences r on } \\
\text { positive } \\
\text { development }\end{array}$ & Yun Chang & $\begin{array}{l}\text { Trabalho submetido ao } \\
\text { corpo docente da } \\
\text { University Graduate } \\
\text { School em } \\
\text { cumprimento parcial } \\
\text { dos requisitos para o } \\
\text { grau de Doutor em } \\
\text { Filosofia na School of }\end{array}$ & $\begin{array}{l}\text { Examinar o impacto do } \\
\text { componente } \\
\text { Aluno curricular } \\
\text { Experiências (ASE) do } \\
\text { curso específico de } \\
\text { programação } \\
\text { Adventure Outdoor } \\
\text { (OAE) na promoção da }\end{array}$ & $\begin{array}{l}\text { Os resultados da análise indicaram } \\
\text { que o programa OAE pode ser } \\
\text { eficaz em favorecer a autonomia } \\
\text { dos jovens e o PYD dos } \\
\text { adolescentes. ASE possibilita um } \\
\text { "reforço de aprendizagem" para os } \\
\text { adolescentes e isso reforça os } \\
\text { resultados de desenvolvimento que } \\
\text { tiveram no início do curso. }\end{array}$ \\
\hline
\end{tabular}


Revista Ibero- Americana de Humanidades, Ciências e Educação- REASE $\quad$ open $\boldsymbol{\sigma}^{\text {access }}$

\begin{tabular}{|c|c|c|c|c|c|c|}
\hline & & & & $\begin{array}{l}\text { Public Health Indiana } \\
\text { University }\end{array}$ & $\begin{array}{l}\text { juventude, incluindo } \\
\text { autonomia e PYD. }\end{array}$ & \\
\hline 17 & 2017 & $\begin{array}{l}\text { Positive Youth } \\
\text { Development Among } \\
\text { Minority Youth: A } \\
\text { Relational } \\
\text { Developmental } \\
\text { Systems Model }\end{array}$ & $\begin{array}{l}\text { Richard M. Lerner, } \\
\text { Jun Wang, Rachel } \\
\text { M. Hershberg, } \\
\text { Mary H. } \\
\text { Buckingham, Elise } \\
\text { M. Harris, } \\
\text { Jonathan } \\
\text { Tirrell, Edmond P. } \\
\text { Bowers r and } \\
\text { Jacqueline } \\
\text { Lerner. }\end{array}$ & $\begin{array}{l}\text { N.J. Cabrera and B. } \\
\text { Leyendecker (eds.), } \\
\text { Handbook on Positive } \\
\text { Development of } \\
\text { Minority Children } \\
\text { and Youth. }\end{array}$ & $\begin{array}{l}\text { Apresentar a visão geral do } \\
\text { desenvolvimento positivo } \\
\text { da juventude (PYD) na } \\
\text { perspectiva dos sistemas de } \\
\text { desenvolvimento relacional } \\
\text { e subjetivo (RDS) e discutir } \\
\text { como o sucesso PYD pode } \\
\text { ser promovido entre os } \\
\text { diversos e diferentes jovens } \\
\text { da América. }\end{array}$ & $\begin{array}{l}\text { Os resultados mostraram que há } \\
\text { limitações nas amostras de } \\
\text { pesquisa PYD, há ausência de uma } \\
\text { amostra representativa de jovens } \\
\text { de minorias nessa pesquisa. } \\
\text { Existem implicações na aplicação } \\
\text { de programas PYD que visam } \\
\text { promover o sucesso entre os jovens } \\
\text { das minorias nos EUA e } \\
\text { internacionalmente. }\end{array}$ \\
\hline 18 & 2017 & $\begin{array}{lr}\text { Commentary } & \text { on } \\
\text { Cross-Cultural } & \\
\text { Perspectives } & \text { on } \\
\text { Positive } & \text { Youth } \\
\text { Development } & \text { With } \\
\text { Implications } & \text { for } \\
\text { Intervention Research }\end{array}$ & $\begin{array}{l}\text { Silvia H. Koller, } \\
\text { Suman Verma. }\end{array}$ & $\begin{array}{l}\text { Child Development } \\
2017 \text { Society for } \\
\text { Research in Child } \\
\text { Development, }\end{array}$ & $\begin{array}{l}\text { Avaliar alguns dos temas } \\
\text { transculturais } \\
\text { implicações PYD com } \\
\text { investigar como a ciência de } \\
\text { intervenção pode beneficiar } \\
\text { ao incorporar uma } \\
\text { abordagem PYD. }\end{array}$ & $\begin{array}{l}\text { Os resultados revelaram que os } \\
\text { jovens engajados em contextos que } \\
\text { proporcionam recursos de outras } \\
\text { pessoas significativas não só eram } \\
\text { menos propensos a exibir } \\
\text { resultados negativos, mas também } \\
\text { tinham maior probabilidade de } \\
\text { apresentar evidências de } \\
\text { desenvolvimento positivo. }\end{array}$ \\
\hline 19 & 2017 & $\begin{array}{l}\text { The five-factor model } \\
\text { for leisure } \\
\text { management: } \\
\text { pedagogies for } \\
\text { assessing personality } \\
\text { differences in positive } \\
\text { youth development } \\
\text { programmes }\end{array}$ & Andrea Anderson & World Leisure Journal & $\begin{array}{l}\text { Fornecer uma visão geral do } \\
\text { lazer e da literatura PYD; } \\
\text { abordar } 33 \text { papel dos cinco } \\
\text { fatores modelo de } \\
\text { personalidade em programas } \\
\text { de lazer e PYD; e sugerir } \\
\text { propostas sobre aplicações } \\
\text { práticas. }\end{array}$ & $\begin{array}{l}\text { Os resultados permitiram verificar } \\
\text { que fornecer uma compreensão } \\
\text { mais clara da personalidade de } \\
\text { alguém não significa estagnação, } \\
\text { ou seja, quanto mais clareza das } \\
\text { diferenças de personalidade em } \\
\text { termos de pontos fortes e fracos, os } \\
\text { sujeitos podem preparar os jovens } \\
\text { para variadas situações que } \\
\text { encontrarão na realidade, fora da } \\
\text { segurança de seu contexto de lazer. }\end{array}$ \\
\hline
\end{tabular}




\begin{tabular}{|c|c|c|c|c|c|c|}
\hline \multicolumn{7}{|c|}{ Continua } \\
\hline 20 & 2017 & $\begin{array}{l}\text { From Theory to } \\
\text { Practice: a critical } \\
\text { review of positive } \\
\text { youth development } \\
\text { program Frameworks }\end{array}$ & $\begin{array}{lr}\text { Mary } & \text { Elizabeth } \\
\text { Arnold, } & \text { Bem } \\
\text { Silliman. } & \end{array}$ & $\begin{array}{l}\text { JYD - Journal of } \\
\text { Youth Development }\end{array}$ & $\begin{array}{l}\text { Revisar a literatura das } \\
\text { estruturas dos programas de } \\
\text { desenvolvimento positivo de } \\
\text { jovens. }\end{array}$ & $\begin{array}{l}\text { Os resultados apontaram que a } \\
\text { lacuna que existe entre o trabalho } \\
\text { dos cientistas pesquisadores do } \\
\text { PYD e aqueles que implementam o } \\
\text { programa PYD gera uma aplicação } \\
\text { irregular e, em alguns casos, } \\
\text { incorreta. Ficou evidente que os } \\
\text { programas locais são mais } \\
\text { predispostos a se basear em alguns } \\
\text { princípios PYD (por exemplo, } \\
\text { conexões com adultos atenciosos, } \\
\text { trabalho de projeto autodirigido, } \\
\text { orientado para jovens lideranças) } \\
\text { do que a aplicação sistemática de } \\
\text { uma estrutura PYD. }\end{array}$ \\
\hline $2, I$ & 2016 & $\begin{array}{l}\text { The Modeling of } \\
\text { School Climate } \\
\text { Perception and } \\
\text { Positiver Youth } \\
\text { Development with } \\
\text { Academic Buoyancy }\end{array}$ & $\begin{array}{l}\text { Farah Bakhshaee, } \\
\text { Elaher Hejazi, } \\
\text { Fariborz Dortaj, } \\
\text { Valiollah Farzad. }\end{array}$ & $\begin{array}{l}\text { Journal of current } \\
\text { research in science }\end{array}$ & 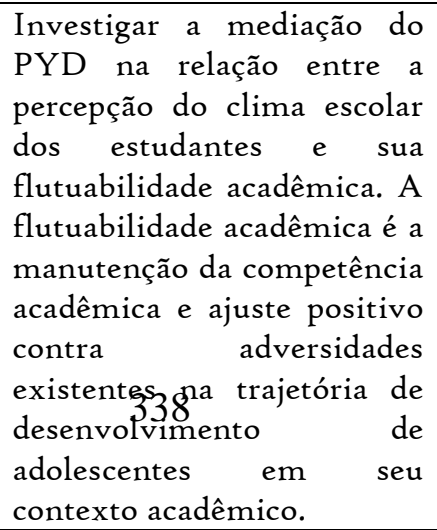 & $\begin{array}{l}\text { Os resultados indicaram que o } \\
\text { efeito da percepção do clima } \\
\text { escolar e do desenvolvimento } \\
\text { positivo dos jovens na } \\
\text { flutuabilidade acadêmica é positivo } \\
\text { e expressivo através do PYD. }\end{array}$ \\
\hline
\end{tabular}


Revista Ibero- Americana de Humanidades, Ciências e Educação- REASE $\quad$ open $\bigodot$ access

\begin{tabular}{|c|c|c|c|c|c|c|}
\hline 22 & 2016 & $\begin{array}{l}\text { The contribution of } \\
\text { urban 4-h positive } \\
\text { youth development } \\
\text { towards social capital } \\
\text { and the implications } \\
\text { for social justice }\end{array}$ & Nia Imani Fields & $\begin{array}{l}\text { Department of } \\
\text { Advanced Studies, } \\
\text { Leadership \& Policy }\end{array}$ & $\begin{array}{l}\text { Analisar as perspectivas dos } \\
\text { educadores urbanos } 4-\mathrm{H} \\
\text { PYD de seus programas e } \\
\text { práticas para servir a jovens } \\
\text { marginalizados; determinar } \\
\text { como eles se encaixam em } \\
\text { uma estrutura de capital } \\
\text { social e; identificar temas } \\
\text { que podem levar à } \\
\text { operacionalização } \\
\text { práticas eficazes. }\end{array}$ & $\begin{array}{l}\text { Os resultados indicaram que } \\
\text { existem iniciativas } 4-\mathrm{H} \text { nacionais, } \\
\text { estaduais e locais para alcançar } \\
\text { jovens marginalizados. Entretanto, } \\
\text { mesmo com maiores esforços de } 4^{-} \\
\mathrm{H} \text { em comunidades de áreas } \\
\text { urbanas e uma crescente população } \\
\text { diversificada, tem havido barreiras } \\
\text { que têm impedido o } 4-\mathrm{H} \text { de servir } \\
\text { adequadamente e / ou } \\
\text { consistentemente aos jovens } \\
\text { marginalizados. }\end{array}$ \\
\hline 23 & 2016 & $\begin{array}{l}\text { Recursos que } \\
\text { contribuyen al } \\
\text { desarrollo positivo en } \\
\text { jóvenes Resources that } \\
\text { promote positive } \\
\text { youth development }\end{array}$ & $\begin{array}{l}\text { Martha } \quad \text { Frías } \\
\text { Armenta e Melanie } \\
\text { Itsel } \quad \text { Barrios } \\
\text { Gaxiola. }\end{array}$ & Escritos de Psicología & $\begin{array}{l}\text { Analisar os recursos que } \\
\text { promovem } \\
\text { desenvolvimento positivo } \\
\text { jovem. }\end{array}$ & $\begin{array}{l}\text { Os resultados confirmaram que } \\
\text { existe uma relação direta dos } \\
\text { recursos psicológicos e sociais com } \\
\text { o desenvolvimento positivo, bem } \\
\text { como os recursos sociais afetam os } \\
\text { psicológicos; apoiando as teorias } \\
\text { analisadas. Não foram encontradas } \\
\text { relações significativas com } \\
\text { recursos econômicos e } \\
\text { desenvolvimento e vibrações } \\
\text { positivas do jovem. }\end{array}$ \\
\hline \multicolumn{7}{|l|}{ Continua } \\
\hline 24 & 2016 & $\begin{array}{l}\text { Intersectional } \\
\text { scholarship \& positive } \\
\text { youth development: } \\
\text { post-modern } \\
\text { paradigm shift in } \\
\text { understanding } \\
\text { adjustment of } \\
\text { marginalized youth }\end{array}$ & $\begin{array}{l}\text { J.-M. Gonzalez, D. } \\
\text { Ştefănel, Radosveta } \\
\text { Dimitrova. }\end{array}$ & $\begin{array}{l}\text { The Central and } \\
\text { Eastern European } \\
\text { Online Library }\end{array}$ & $\begin{array}{l}\text { Explicar339e ampliar a } \\
\text { utilização da metodologia da } \\
\text { interseccionalidade nos } \\
\text { estudos PYD. }\end{array}$ & $\begin{array}{l}\text { Os resultados do estudo revelaram } \\
\text { que reações maternas e paternas } \\
\text { negativas à divulgação de jovens } \\
\text { negros sobre sua identidade de } \\
\text { minoria sexual levava } \\
\text { significativamente o aumento de } \\
\text { comportamentos problemáticos } \\
\text { para todos os jovens. Além disso, } \\
\text { jovens negros americanos também } \\
\text { eram menos propensos do que os } \\
\text { jovens brancos americanos a ter } \\
\text { comportamentos problemáticos se }\end{array}$ \\
\hline
\end{tabular}


Revista Ibero- Americana de Humanidades, Ciências e Educação- REASE $\quad$ open $○$ Access

\begin{tabular}{|c|c|c|c|c|c|c|}
\hline & & & & & & $\begin{array}{l}\text { familiares apoiadores estavam } \\
\text { presentes nessa divulgação. }\end{array}$ \\
\hline 25 & 2016 & $\begin{array}{l}\text { What is positive } \\
\text { youth development } \\
\text { and how might it } \\
\text { reduce substance use } \\
\text { and violence? A } \\
\text { systematic review and } \\
\text { synthesis of } \\
\text { theoretical literature }\end{array}$ & $\begin{array}{l}\text { Chris Bonell, Kate } \\
\text { Hinds, Kelly } \\
\text { Dickson, James } \\
\text { Thomas, Adam } \\
\text { Fletcher, Simon } \\
\text { Murphy, G. J. } \\
\text { Melendez-Torres, } \\
\text { Carys Bonell e } \\
\text { Rona Campbell. }\end{array}$ & $\begin{array}{l}\text { Bonell et al., BMC } \\
\text { Public Health }\end{array}$ & $\begin{array}{l}\text { Sistematizar literaturas } \\
\text { teóricas que abordam o PYD } \\
\text { e como isso pode reduzir o } \\
\text { uso de substâncias e } \\
\text { violência, bem como } \\
\text { examinar o processo e os } \\
\text { resultados das intervenções } \\
\text { do PYD. }\end{array}$ & $\begin{array}{l}\text { Os resultados revelaram i6 estudos que } \\
\text { sintetizam que o PYD visa } \\
\text { proporcionar aos jovens } \\
\text { relacionamentos afetivos e } \\
\text { experiências diversas que possibilitem } \\
\text { o seu desenvolvimento de } \\
\text { autorregulação e múltiplos ativos } \\
\text { positivos. Estes, por sua vez, protegem } \\
\text { ou compensam o envolvimento no uso } \\
\text { de substâncias e violência. Verificou-se } \\
\text { que os estudos existentes não } \\
\text { esclarecem como a auto regulação } \\
\text { intencional é desenvolvida e quais } \\
\text { aspectos positivos específicos ativos de } \\
\text { proteção contra o uso de substâncias ou } \\
\text { violência. }\end{array}$ \\
\hline 26 & 2016 & $\begin{array}{l}\text { Exploring the Long- } \\
\text { Term Impact of a } \\
\text { Positive Youth } \\
\text { Development-Based } \\
\text { Alcohol, Tobacco and } \\
\text { Other Drug } \\
\text { Prevention Program }\end{array}$ & $\begin{array}{lr}\text { Rebecca Wade- } \\
\text { Mdivanian, Dawn } \\
\text { Anderson-Butcher, } \\
\text { Tarkington J. } \\
\text { Newman } \\
\text { Danielle } \\
\text { Ruderman, E. Jill } \\
\text { Smock \& Stephanie } \\
\text { Christie. }\end{array}$ & $\begin{array}{l}\text { Journal of Alcohol \& } \\
\text { Drug Education são } \\
\text { propriedade da } \\
\text { American Alcohol } \\
\text { \&Drug Information } \\
\text { Foundation }\end{array}$ & $\begin{array}{l}\text { Avaliar o impacto de um } \\
\text { programa de prevenção de } \\
\text { substâncias em PYD } \\
\text { mediante o programa } \\
\text { Juventude para Juventude } \\
\text { (Y2Y) Internacional. } \\
\qquad 340\end{array}$ & $\begin{array}{l}\text { Os resultados mostraram melhorias } \\
\text { significativas do pré ao pós- } \\
\text { conferência sobre o conhecimento dos } \\
\text { riscos do álcool, tabaco e outras drogas } \\
\text { (ATOD), atitudes em relação ao uso, } \\
\text { autoeficácia, percepções de liderança e } \\
\text { participação futura e envolvimento no } \\
\text { programa Y2Y. As descobertas deste } \\
\text { estudo mostram a importância de } \\
\text { programas de prevenção como } Y_{2} Y \text { no } \\
\text { apoio positivo do desenvolvimento da } \\
\text { juventude }\end{array}$ \\
\hline 27 & 2016 & $\begin{array}{l}\text { Developing a Federal } \\
\text { Research Agenda for } \\
\text { Positive } \\
\text { Development: } \\
\text { Identifying Gaps in the } \\
\begin{array}{l}\text { Field and an Effective } \\
\text { Consensus } \\
\text { Approach Building }\end{array} \\
\end{array}$ & $\begin{array}{l}\text { Allison B. Dymnicki, } \\
\text { Suzanne Le } \\
\text { Menestrel, Michelle J. } \\
\text { Boyd, Lisa Lauxman, } \\
\text { Sarah E. Oberlander, } \\
\text { David M. Osher. }\end{array}$ & $\begin{array}{l}\text { Journal of Youth } \\
\text { Development - Bridging } \\
\text { Research and Practice }\end{array}$ & $\begin{array}{l}\text { Apresentar estudos consensuais } \\
\text { com representantes de i6 } \\
\text { agências federais para } \\
\text { desenvolver uma agenda de } \\
\text { pesquisa focada em PYD. }\end{array}$ & $\begin{array}{l}\text { Os resultados apontaram que existem } \\
\text { muitas áreas específicas para pesquisas } \\
\text { futuras em PYD e permitiu compilar } \\
\text { uma descrição das implicações futuras } \\
\text { para a política de PYD e colaborações } \\
\text { interagências. }\end{array}$ \\
\hline
\end{tabular}


2 Discussão

O presente artigo objetivou realizar uma revisão integrativa da literatura que considera os recursos desenvolvimentais e o desenvolvimento juvenil positivo, mais conhecido internacionalmente como Positive Youth Development (PYD).

Então, o artigo de Etteka e Agans (2020) já observa a influência da Pandemia COVID-ı́ nesse processo de desenvolvimento juvenil. Aqui, identificam-se desafios e sugerem-se ideias cooperativas de lazer para eficácia dos programas PYD submetidos às medidas de distanciamento.

Ainda, sobre aspectos da área do lazer, Andrea Anderson (2017) afirma que as práticas corretas de lazer proporcionam uma experiência mais positiva e prepara os jovens para variedades de situações que encontrarão no mundo real, fora da segurança de seu contexto de lazer.

Deveras, isso é demonstrado quando Andrea Vest Etteka e Jennifer P. Agans (2020) apontam que há riscos de negligenciar o sentido do lazer durante a pandemia porque para muitos jovens pode ser tempo livre o tempo todo e isso impacta imensamente no seu desenvolvimento.

Não somente o lazer, mas também é interessante destacar a criatividade nesse processo de desenvolvimento juvenil, sendo imprescindível para a educação, principalmente na adolescência (Mézerville, 2019).

Segundo Claire de Mézerville (2019), a criatividade é uma habilidade para autorrealização e engajamento positivo na sociedade, trata-se de uma manifestação de autonomia.

Em vista disso, a promoção do PYD exige disposições escolares e adultos comprometidos, bem como, cenários que impulsionem jovens a se envolverem, a criarem e a progredirem (Mézerville, 2019).

Além disso, essa pesquisa permitiu identificar artigos que trazem a questão contextual para a discussão da realidade do Desenvolvimento Positivo Jovem em países de baixa e média renda, conforme Olenik (2018).

Dito isso, Kristy Allen (2018) reflete sobre a promoção do bem-estar juvenil considerando as iniciativas de engajamento, utilizadas nas estruturas do Desenvolvimento Positivo Jovem, sem deixar de valorizar também o contexto e, nesse caso, canadense.

Igualmente, Franco e Rodrigues (2018) contribuem com essa proposta contextual quando relacionam as crenças de autoeficácia e recursos desenvolvimentais em diferentes esferas sociais, sinalizando a necessidade de maior investigação da temática no contexto brasileiro.

Também, Michael Sieng, Scott Cloutier e Katherine Irimata (2018) afirmam, ao avaliarem programas PYD, que a sistematização para qualificar não se aplica a todos os jovens em todos os países por conta da diversidade cultural e particularidades de cada programa PYD. 
Nesse sentido, podem existir limitações nas amostras de pesquisa dos participantes no Desenvolvimento Positivo Jovem e isso implica também na utilização do PYD em outros contextos como afirma Lerner et al. (2017).

Por exemplo, a ausência de número suficiente de representação jovem de diversas raças e etnias, grupos e de configurações urbanas limita o conjunto de dados do estudo $4-\mathrm{H}$ (Lerner et al., 2017).

Dessa forma, esse modelo de Lerner restringe saber como é o desenvolvimento positivo da juventude para a juventude minoritária da América (Lerner et al., 2017).

Por certo, no que concerne ainda sobre limitações PYD, Geldhofa et al. (2019) defendem que pessoas que são ricas em cuidados, sobretudo, com os outros, não são necessariamente 'bem adaptadas', pois cuidado excessivo pode ser mal-adaptativo.

Correlativamente, lacunas das estruturas PYD também são identificadas por Arnold e Silliman (2017). A crítica mira-se na relação do trabalho dos cientistas pesquisadores e praticantes do programa, pois alguns princípios sobressaem-se à aplicação sistemática de uma estrutura PYD.

Similarmente, Shek, Dou, Zhu e Chai (2019) registram que há uma conexão incompleta entre diferentes temas e domínios no PYD, a ausência de integração entre os processos, filosofias e programas do PYD causa incerteza na implementação do PYD.

Contudo, mesmo com as possíveis limitações identificadas nos programas PYD, Fields 342 (2016) assegura que há iniciativas eficazes para jovens marginalizados, principalmente em comunidades urbanas e diversificadas.

Ademais, Koller e Verma (2017) defendem que jovens envolvidos em contextos que proporcionam recursos e pessoas significativas são menos propensos a comportamentos negativos, como também têm mais probabilidade de mostrar evidências de PYD.

Não somente isso, como é possível identificar que a manutenção da competência acadêmica e ajuste positivo contra adversidades existentes na trajetória do jovem é mediada pelo $\mathrm{PYD}$, segundo Bakhshaee, Hejazi, Dortaj, Farzad (2016).

Tal qual, o trabalho de Kramer, Wilcox e Lawson (2020) ratifica em loco o uso das práticas de PYD como recurso de melhoria escolar com estratégias para facilitar o engajamento dos estudantes, agenciando e desenvolvendo a identidade.

Além disso, Yun Chang (2017) através de uma pesquisa empírica realiza um caminho que discute sobre o impacto de atividades do componente curricular Aluno Autônomo Experiências (ASE) que pode ser uma ferramenta eficaz na promoção do PYD.

Porquanto, conjuntos de valores (cooperação, caráter, compaixão) e contextos favoráveis (família, escola e comunidade), aqueles recursos psicológicos e esses sociais têm relação direta com o PYD, comprovação dos resultados da pesquisa de Armenta e Gaxiola (2016). 
Como afirmam Bonell et al. (2016), o PYD busca proporcionar aos jovens relacionamentos afetivos e experiências diversas que possibilitem o seu desenvolvimento de autorregulação, autonomia e múltiplos ativos positivos.

Por isso, denota-se que tais arranjos que priorizam a afetividade protegem ou compensam do envolvimento no uso de substâncias e da prática de violência, tal qual afirma Bonell et al. (2016).

Ainda, essas informações dialogam com os resultados dos estudos de Wade-Mdivanian et al. (2016), os quais mostram avanços nas conferências referentes aos riscos do álcool, tabaco e outras drogas inseridas num programa PYD.

Nessa perspectiva, é possível também analisar nas discussões e resultados dos artigos aqui catalogados que o apego dos pais e dos pares colaboram para fatores do desenvolvimento positivo da juventude para Mohamed, Hamzah e Samah (2017).

Logo, Armenta e Gaxiola (2016) confirmam o argumento de que tanto recursos psicológicos quanto sociais impactam diretamente no desenvolvimento positivo do jovem individualmente e de suas famílias e comunidades.

Assim, a socialização impacta no desenvolvimento da identidade juvenil, mesmo em diferentes ambientes sociais. Com efeito, a eficiência das ações depende muito do conhecimento e experiência adquiridos e da avaliação (Nikitorowicz, Sawicki, Zylkiewicz-Plonska, 2017).

Portanto, os programas PYD influenciam comportamentos dos jovens, inclusive pelas relações de interseccionalidade, conforme Gonzalez, Ştefănel e Dimitrova (2016), e por essa razão citam considerações para futuras pesquisas, aplicações e empreendimentos políticos.

Sendo assim, confirma-se elaboração de agenda de pesquisa PYD no artigo de Dymnicki et al. (2016) onde os formuladores de políticas veem a intervenção e como a pesquisa está sendo alinhada, pois conceitualmente PYD reflete mudança de paradigma.

\section{Conclusão}

Esse trabalho tem o objetivo de compilar literaturas que abordem sobre os recursos desenvolvimentais e o desenvolvimento juvenil positivo, revisando-as e considerando os princípios PYD para também avançar em propostas para o contexto brasileiro.

Diante disso, os recursos desenvolvimentais e PYD relacionam-se entre si, visto que, em todos os artigos integrados à essa revisão identifica-se ao menos um dos 40 ativos de desenvolvimento distribuídos em oito categorias do modelo de Benson.

Desse modo, considera-se que ativos externos do modelo de Benson como lazer, nível econômico do país, estrutura escolar, diversidade cultural, vínculos familiares, políticas públicas de apoio às juventudes são mecanismos do PYD. 
Ainda, baseando-se no modelo de Benson, há também recursos internos como criatividade, autoeficácia, auto-regulação, dentre outros, citados nos artigos dessa revisão, que se atrelam à juventude e associam-se aos programas PYD.

Por conseguinte, nessa revisão fica evidente que a juventude possui forças e recursos que e quando cercada de contextos que favoreçam oportunidades socioeconômicas e relações afetivas benéficas tem-se comportamentos positivos e bem-estar, propostas intrínsecas ao PYD.

Enfim, esse levantamento de literatura possibilitou identificar bases teóricas referentes aos recursos desenvolvimentais e o PYD e também observar lacunas que podem estimular novas pesquisas e evidências cientificas que promovam o bem-estar da juventude no Brasil.

\section{Referências Bibliográficas}

Agência Brasil [homepage na internet]. Um terço dos jovens do mundo vive em situação de vulnerabilidade social. Disponível em: <https://agenciabrasil.ebc.com.br $\geq$. Acesso em: or dez 2020.

ALLEN, Kristy. Understanding the impact of youth engagement on positive youth development. Dissertação (Mestrado em Saúde Pública) - University of Guelph, Faculty of Health Sciences; 2018.

ANDERSON, Andrea. The five-factor model for leisure management: pedagogies for assessing personality differences in positive youth development programmes. World Leisure Journal, v. 59, n.I, p. 70-76, 2017.

ARMENTA, Martha Frias; GAXIOLA, Melanie. Recursos que contribuyen al desarrollo positivo en jóvenes Resources that promote positive youth development. Escritos de Psicología, v. 9, n.3, p. 37-44, 2016.

ARNOLD, Mary Elizabeth; SILLIMAN, Ben. From Theory to Practice: a critical review of positive youth development program Frameworks. JYD - Journal of Youth Development, v. I2, 2017.

BAKHSHAEE, Farah; HEJAZI, Elahe; DORTAJ, Fariborz; FARZAD, Valiollah. The Modeling of School Climate Perception and Positive Youth Development with Academic Buoyancy. J. Curr. Res. Sci., v. I, p. 94-10o, 2016.

BONELL, Chris; HINDS, Kate; DICKSON, Kelly; THOMAS, James; FLETCHER, Adam; MURPHY, Simon; MELENDEZ-TORRES, G. J.; BONELL, Carys e CAMPBELL, Rona. What is positive youth development and how might it reduce substance use and violence? A systematic review and synthesis of theoretical literature. BMC Public Health, 2016.

Chang, Yung. Exploring the effect of autonomous student experiences on positive youth development. Tese (Doutorado em Filosofia) - University Graduate School, School of Public Health Indiana University; 2017.

DUTRA-THOMÉ, Luciana; LEME, Vanessa Barbosa Romera; PEREIRA, Anderson Siqueira; DIAS, Ana Cristina Garcia; KOLLER, Silvia Helena; ALBUQUERQUE Emily Souza Gaião e. 
Fatores protetivos e de risco na transição para a vida adulta nas cinco regiões brasileiras. Avances en Psicología Latinoamericana, v. 35, n.3, 2017.

DUTRA-THOMÉ, Luciana; PEREIRA, Anderson Siqueira; KOLLER, Silvia Helena. Habilidades sociais e fatores de risco e proteção na adultez emergente. Porto Alegre, Psico, v. 47 n. 4, p. 268-278, 2016.

DYMNICKI, Alisson B.; MENESTREL, Suzanne Le; BOYD, Michelle J.; LAUXMAN, Lisa; OBERLANDER, Sarah E.; OSHER, David M.; Developing a Federal Research Agenda for Positive Youth Development: Identifying Gaps in the Field and an Effective Consensus Building Approach. Journal of Youth Development - Bridging Research and Practice, v. II, n. oI, 2016.

EICHAS, Kyle; WREDER, Laura Ferrer; OLSSON, Tina M. Contributions of Positive Youth Development to Intervention Science. Child \& Youth Care Forum, 2019.

ETTEKA, Andrea Vest; AGANS, Jennifer P. Positive Youth Development Through Leisure: Confronting the COVID-i9 Pandemic. Journal of Youth Development, v. 15, 2020.

FIELDS, Nia Imani. The contribution of urban $4^{-h}$ positive youth development towards social capital and the implications for social justice. JOE, v. 55, n. 6, 2016.

FRANCO, Gisele de Rezende; RODRIGUES, Marisa Cosenza. Autoeficácia e Desenvolvimento Positivo dos Jovens: Uma Revisão Narrativa da Literatura. Ribeirão Preto: Trends Psychol, v. 26, n. 4, p. 2267-2282, 2018

GELDHOFA, G. John; LARSENB, Torill; URKEB, Helga, HOLSENB, Ingrid; LEWISA, Hillary; TYLERA, Corine P. Indicators of positive youth development can be maladaptive: The example case of caring. Journal of Adolescence 2019; 71: I-9.

GONZALEZ, J. M.; ŞTEFĂNEL, D.; DIMITROVA, Radosveta. Intersectional scholarship \& positive youth development: post-modern paradigm shift in understanding adjustment of marginalized youth. The Central and Eastern European Online Library, v. 9, n. 58, p. 2, 2016.

HAMILTON, S. F.; HAMILTON, M. A.; PITTMAN, K. Principles for Youth Development. In S.F. HAMILTON \& M.A. HAMILTON (Eds.) 2004, The Youth Development Handbook: Coming of Age in American Communities. Thousand Oaks: Sage Publications, Inc., p.3-22.

KOLLER, Silvia Helena; VERMA, Suman. Commentary on Cross-Cultural Perspectives on Positive Youth Development With Implications for Intervention Research. Child Development, p. I-5, 2017.

KRAMER, Catherine S; WILCOX, Kristen C.; LAWSON, Hal A. Positive youth development as an improvement resource in odds-beating secondary schools. Preventing School Failure: Alternative Education for Children and Youth, 2020.

LERNER, Richard M.; JUN Wang; HERSHBERG, Rachel M.; BUCKINGHAM, Mary H.; HARRIS, Elise M.; TIRRELL, Jonathan M.; BOWERS, Edmond P.; LERNER, Jacqueline V. Positive Youth Development Among Minority Youth: A Relational Developmental Systems Model. Handbook on Positive Development of Minority Children and Youth 2017. 
MAIA, Joviane Marcondelli Dias; WILLIAMS, Lucia Cavalcanti de Albuquerque. Fatores de risco e fatores de proteção ao desenvolvimento infantil: uma revisão da área. Temas em Psicologia, v.i3, n. 2, p. 91-103, 2005.

MÉZERVILLE, Claire de. Adolescence and creativity: cognitions and affect involved in positive youth development. Arts and Humanities Open Access Journal, v. 3, p. 163-168, 2019.

MOHAMED, Nor Hidayah; HAMZAH, Siti Raba'ah; SAMAH, Ismi Arif Ismail Bahaman Abu. Parental and Peer Attachment and its Relationship with Positive Youth Development. International Journal of Academic Research in Business and Social Sciences, v. 7, n. 9, p. 2222-6990, 2017.

NIKITOROWICZ, Jerzy; SAWICKI, Krzysztof; ZYLKIEWICZ-PLONSKA, Emilia. Youth identity from ecological perspective between education and prevention. Society. Integration. Education, v. 3, p. 243-254, 2017.

NORZE, Jeantyl; CATER, Melissa. A Systematic Review of Program Quality in the Field of Positive Youth Development. Advances in Sciences and Humanities - Science Publishing Group, v. 6, n. 2, p.58-69, 2020.

OLENIK, Christina. The Evolution of Positive Youth Development as a Key International Development Approach. Global Social Welfare, 2018.

SARIZADEH, Mohammad Sadegh; NAJAFI, Mahmoud; REZAEI, Ali Mohammad. The prediction of depression based on religious coping and the components of positive youth development in adolescentes. Mental Health, Religion \& Cultur, 2020.

SHEK, Daniel TL; DOU, Diya; ZHU, Xiaoqin; CHAI, Wenyu. Positive youth development: current perspectives. Department of Applied Social Sciences, The Hong Kong Polytechnic University, Hong Kong, People's Republic of China. Adolescent Health, Medicine and Therapeutics, v. IO, p. I3I-I4I, 2019.

SIENG, Michael; ClOUTIER, Scott; IRIMATA, Katherine. Positive Youth Development Sustainability Scale (PYDSS): The Development of an Assessment Tool. Journal of Social Change, v. ro, p. 81-97, 2018.

SOARES, Ana; PAIS-RIBEIRO, José L.; SILVA, Isabel. Recursos do Desenvolvimento na Adolescência - Revisão Integrativa. Facultad de Psicología - Universidad Católica del Uruguay: Ciencias Psicológicas, v. I2, n. I, 2018.

WADE-MDIVANIAN, Rebecca; ANDERSON-BUTCHER, Dawn; NEWMAN, Tarkington J.; RUDERMAN, Danielle E.; SMOCK, Jill; CHRISTIE, Stephanie. Exploring the Long-Term Impact of a Positive Youth Development-Based Alcohol, Tobacco and Other Drug Prevention Program. Journal of Alcohol \& Drug Education, 2016.

WARD, Rolanda L.; CARTER, Tanyetta; SIDDIQ, Nazia. The Role of Positive Youth Development, Educational Policy, and Cultural Relevancy in School Settings. Niagara: Departamento de Serviço Social, Niagara University; 2019. 